\title{
Colonization with heterologous bacteria reprograms a Caenorhabditis elegans nutritional phenotype
}

Authors: Qing Sun $^{1} \uparrow$, Nicole M. Vega ${ }^{2,6} \uparrow$, Bernardo Cervantes ${ }^{3}$, Christopher P. Mancuso ${ }^{4,5}$, Ning Mao ${ }^{3,5,7,8}$, Megan Taylor ${ }^{6}$, James J. Collins ${ }^{1,3,7,8}$, Ahmad S. Khali1 ${ }^{4,5,8}$, Jeff Gore ${ }^{2 *}$, and Timothy K. $\mathrm{Lu}^{1,9,10 *}$.

\section{Affiliations:}

${ }^{1}$ Synthetic Biology Center, MIT, Cambridge, MA 02139, USA.

${ }^{2}$ Department of Physics, MIT, Cambridge, MA 02139, USA.

${ }^{3}$ Institute for Medical Engineering \& Science and Department of Biological Engineering, MIT, Cambridge, MA 02139, USA.

${ }^{4}$ Biological Design Center, Boston University, Boston, MA, USA.

${ }^{5}$ Department of Biomedical Engineering, Boston University, Boston, MA, USA.

${ }^{6}$ Biology Department, Emory University, Atlanta, GA, USA

${ }^{7}$ Broad Institute of MIT and Harvard, Cambridge, MA 02138, USA.

${ }^{9}$ Department of Electrical Engineering and Computer Science, MIT, Cambridge, MA 02139, USA

\footnotetext{
${ }^{10}$ Department of Biological Engineering, MIT, Cambridge, MA 02139, MA.

*Corresponding author. Email: timlu@mit.edu (T.K.L.), gore@mit.edu
}

$\dagger$ These authors contributed equality to this work.

\begin{abstract}
Animals rely on the gut microbiome to process complex food compounds that the host cannot digest and to synthesize nutrients that the host cannot produce. New systems are needed to study how the expanded metabolic capacity provided by the gut microbiome impacts the nutritional status and health of the host. Here we colonized the nematode Caenorhabditis elegans gut with cellulolytic bacteria that enabled $C$. elegans to utilize cellulose, an otherwise indigestible substrate, as a carbon source. The nutritional benefits of colonization with cellulolytic bacteria were assayed directly, by incorporation of isotopic biomass, and indirectly, as host larval yield resulting from glucose release in the gut. As a community component in the worm gut, cellulolytic bacteria can also support additional bacterial species with specialized roles, which we demonstrate by using Lactobacillus to protect against Salmonella infection. As a model system, C. elegans colonized with cellulolytic bacteria can be used to study microbiome-host interactions. Engineered microbiome communities may provide host organisms with novel functions, such as the ability to use more complex nutrient sources and to fight against pathogen infections.
\end{abstract}

One Sentence Summary: Heterologous bacteria colonizing an animal gut help digest complex sugars to provide nutrition for the host in a model system. 
The gut microbiome is a complex community of microbes, the ecology of which is intimately tied to the physiology of its host organism $(1,2)$. These symbiotic microbes have been shown to contribute to the nutritional status and metabolism of the host, train the immune system, and control brain development and behaviors (3). Carbohydrates are important sources of energy for both microbes and animals (4). Simple sugars like glucose and lactose can be easily absorbed by hosts, but more complex carbohydrates and plant polysaccharides, including cellulose, xylans, resistant starch, and inulin, are not as easily digested. The gut microbiome can confer upon its host, such as termites (5) and ruminants (6), caloric benefits by breaking down these ingested plant carbohydrates, which the host enzymes cannot digest (7). The gut microbiome can also evolve to adapt to new carbohydrate sources when hosts change their eating habits. For example, the Japanese, as a result of their diet, have intestinal microbiomes that have gained algal carbohydrate processing enzymes for seaweed processing through horizontal gene transfer from marine bacteria (8). However, research into the use of non-native bacteria to perform nutritional functions for other animal hosts has been limited. An understanding of how the various species of microorganism constituting the microbiome affect their host is fundamental to deriving the potential benefits of manipulating the microbiome.

In this study, we assembled a functional microbiome in a simple animal gut to allow host utilization of a complex carbohydrate source. We used the nematode Caenorhabditis elegans as the animal host because it has been extensively used as a model system to elucidate mechanisms of interaction between prokaryotes and their hosts $(9,10)$. Specifically, we colonized C. elegans with cellulolytic bacteria that break down cellulose in the gut, such that the released glucose is available as a nutrient for both $C$. elegans itself and the colonizing bacteria. To move this system toward higher community complexity as occurs in a natural gut microbiome, we added an additional bacterial species with the specialized function of preventing infection by pathogens. Overall, our results indicate that by assembling a functional community in the $C$. elegans gut, we can extend the nutrition processing and pathogen inhibition capacities of the animal host.

Results:

To introduce a novel carbon processing capability to C. elegans (Fig. 1), we started by selecting cellulose-degrading microbes as potential intestinal colonists. To identify these functional bacteria members, we assembled a collection of $C$. elegans native gut bacteria (11) (see Table $\mathrm{S} 1$ for a full list) and tested these species for their ability to utilize carboxymethyl cellulose (CMC) and other carbon sources by measuring bacterial optical density following in vitro incubation (Fig. S1). Additionally, we checked the cellulose degradation capacity for each strain using a Congo Red assay $(12,13)$. Briefly, after overnight culture on CMC plates and visual confirmation of bacterial growth, we stained the plates with Congo Red. As Congo Red stains glucose polymers, a halo surrounding the colony indicates cellulase activity. None of the native C. elegans isolates (Fig. 2A) exhibited detectable cellulase activity, which reflects their lack of cellulose processing capability. To further identify cellulolytic bacteria, we screened a set of soil bacteria (Table S1) and identified Pseudomonas cellulosa and Bacillus subtilis as cellulase active bacteria (Fig. 2A). Of these strains, $P$. cellulosa produced the highest levels of reducing sugars, including glucose (Fig. 2B), suggesting that this strain could be a potentially useful nutritional endosymbiont for the C. elegans host and supply it with nutrients that would otherwise be unavailable. 
We next sought to determine whether a cellulolytic organism could successfully colonize the worm intestine. $C$. elegans feed on bacteria, a certain percentage of which escape digestion and colonize the gut $(14,15)$. For colonization, we allowed synchronized adult $\mathrm{N} 2$ worms to feed in liquid bacteria culture to ensure that all the worms experienced a uniform environment (15). Worms were then collected, washed, and mechanically disrupted to release intestinal bacteria. These suspensions were then plated on agar to estimate bacterial colonization (Fig. 2C). Naturally cellulolytic bacteria $P$. cellulosa and B. subtilis, as well as a natively non-cellulolytic bacterium, Pseudomonas putida, which had been engineered to produce endoglucanase (see Methods), achieved a colonization density of $\sim 10^{2}-10^{4}$ colony forming units (CFU) per worm (Fig. 2D). Colonization was stable for at least two days in the $C$. elegans gut. All of these bacterial species were therefore able to colonize the host intestine, albeit at different population sizes, but at colonization levels comparable to those of native C. elegans gut strains (Fig. S2).

To determine whether bacteria identified as being cellulolytic in vitro could hydrolyze complex sugars in the gut environment, we pre-colonized C. elegans with the cellulolytic strain P. cellulosa. $P$. cellulosa was chosen for its strong ability to degrade CMC and release reducing sugars, including glucose (Fig. 2B). Since $P$. cellulosa grows to low densities in culture, we used E. coli OP50 as a supplemental food source to ensure that worms had adequate nutrition during growth. Synchronized N2 worms raised on mixed lawns of P. cellulosa and E. coli OP50 on solid NGM medium showed intestinal colonization by $P$. cellulosa as young adults 46 hours post-L1 (Fig. 2D), indicating that this organism is able to colonize the host. Pre-colonized worms were then incubated for 24 hours in liquid media (M9 worm buffer) with: 1) no carbon source, 2) cellulose (in the form

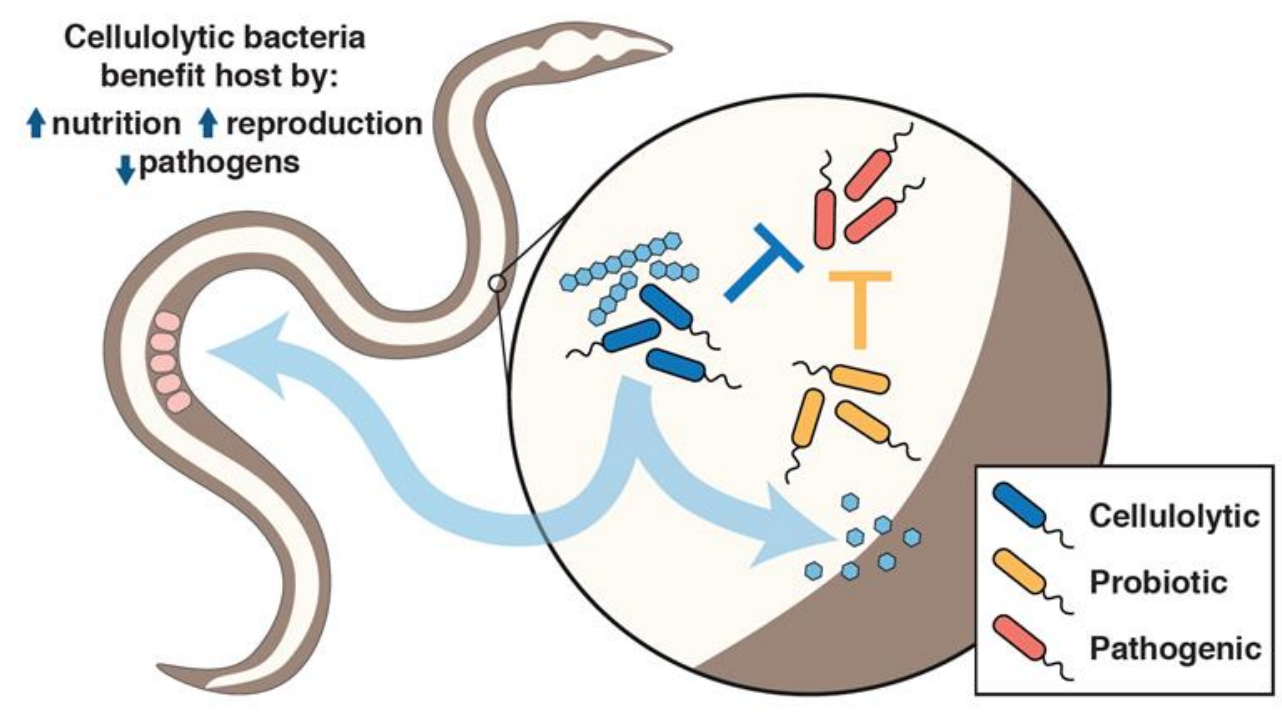

Figure 1. Benefits provided by colonization of the $C$. elegans gut by a heterologous microbial community. Cellulolytic bacteria are able to break down cellulose, such that the released glucose can serve as nutrition for both $C$. elegans and colonized bacteria. Additional bacterial species can improve resistance against pathogenic bacteria. 

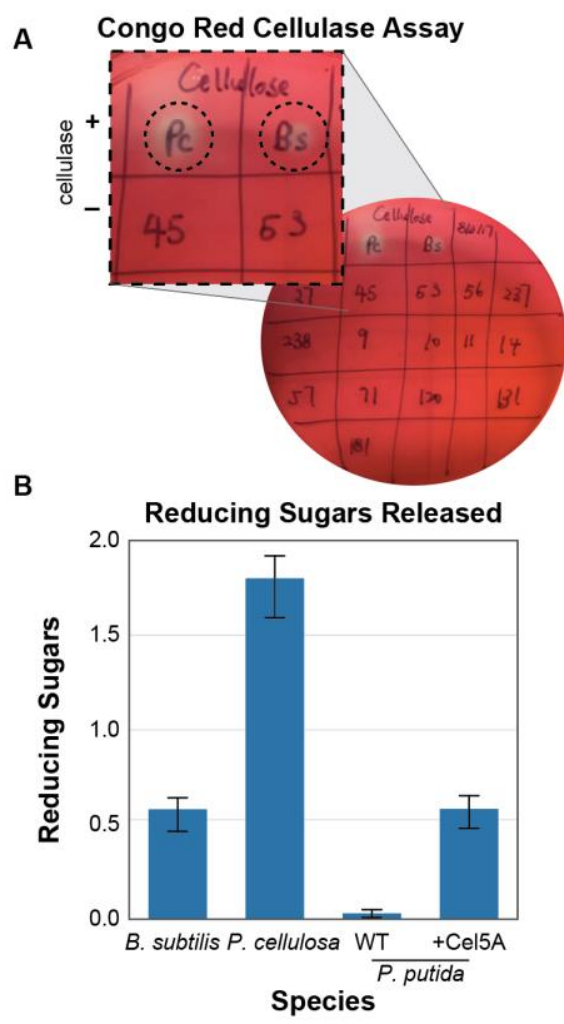

C

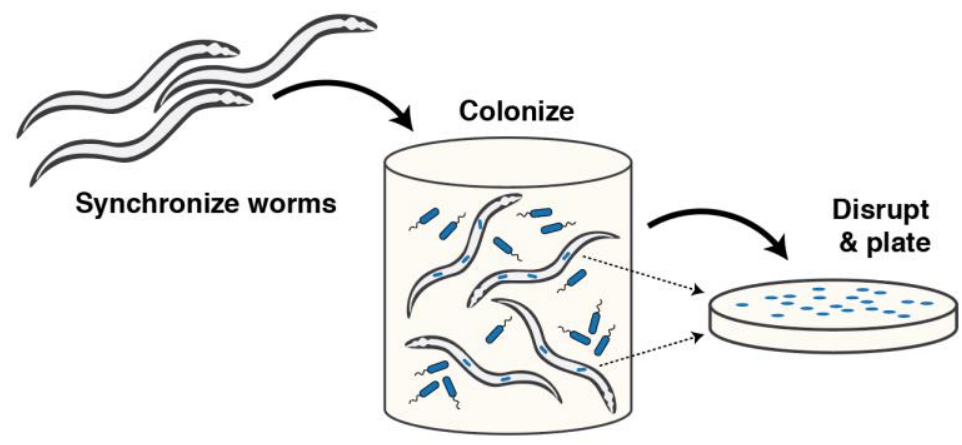

D

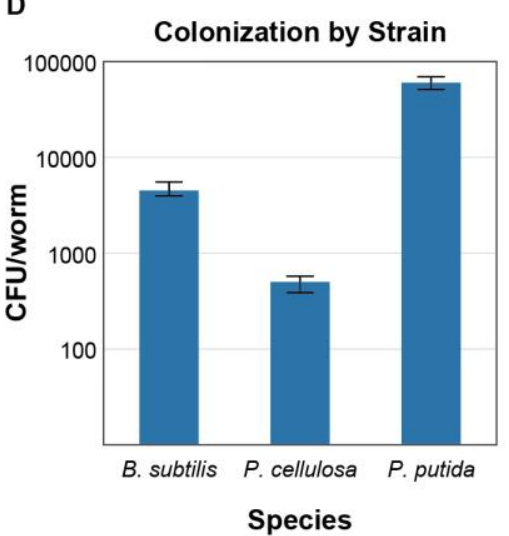

$\mathrm{E}$

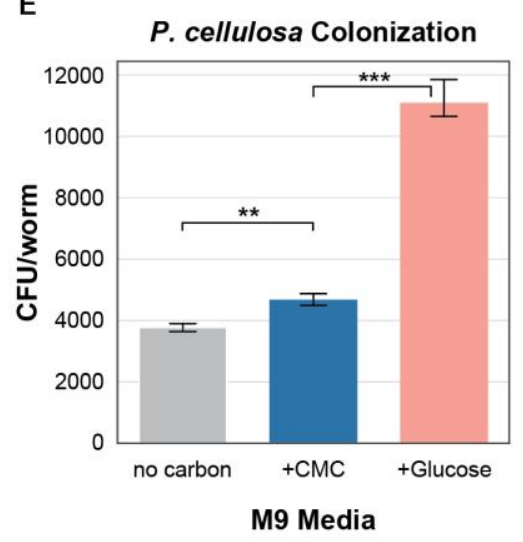

Figure 2. Bacterial colonization and function in worm gut. A) Cellulase functional screening by Congo Red assay. Halos on the plate indicate cellulase activity. Pc: Pseudomonas cellulosa, Bs: Bacillus subtilis. Numbered strains are from the $C$. elegans native gut microbiome as listed in Table S1. B) Production of reducing sugars by cellulose-degrading bacterial strains. C) Schematic of bacterial colonization and analysis procedure. Synchronized L1 wild-type worms were grown on selected strains of live bacteria and then disrupted for colonization analysis by plating and colony counting. D) Colony-forming units (CFU) of various bacterial species in the worm gut. E) C. elegans colonized with cellulolytic $P$. cellulosa and incubated with different carbon sources. $* * \mathrm{P}<0.01$, $* * * \mathrm{P}<0.001$, Student's t test. Error bars represent $95 \%$ confidence intervals of the mean.

of CMC), or 3) glucose. After incubation, worms were washed and digested to check gut bacterial density. We found that M9 buffer with CMC as a carbon source enhanced the colonization density of $P$. cellulosa compared with no carbon source (Fig. 2E). This experiment demonstrated that a cellulolytic bacterial strain, $P$. cellulose, in the $C$. elegans gut hydrolyzed CMC and benefited from the released sugar. Glucose as a carbon source further enhanced colonization density because free sugar monomers are an easier-to-access carbon source than CMC.

Next, we sought to determine whether colonization by cellulolytic bacteria would allow the host to use the otherwise indigestible cellulose carbon source. C. elegans is able to use simple carbohydrates, including glucose (16), but does not produce enzymes that degrade cellulose. We hypothesized that if cellulolytic organisms in the gut could liberate carbon in a form that is available and metabolizable by the host, colonization of the gut with a cellulolytic microbiome would provide this host with the ability to use cellulose as a growth substrate. Again, we chose $P$. cellulosa as the colonizing bacteria. Three approaches were used to investigate whether C. elegans utilized glucose released by gut bacteria. 
First, we used radionucleotide $\left({ }^{14} \mathrm{C}\right)$ incorporation as a direct measurement of cellulose-derived carbon incorporation into worm biomass. Briefly, adult wild-type worms were colonized with $E$. coli OP50 (non-cellulose degrader) or P. cellulosa, then incubated in S medium with heat-killed E. coli $\mathrm{OP} 50$ and trace ${ }^{14} \mathrm{C}$ labeled CMC (radiolabeled cellulose purified from Arabidopsis thaliana) (17) for 24 hours to allow bacterial degradation of the substrate to proceed. After incubation on the radionucleotide substrate, worms were washed and treated with an antibiotic cocktail for 24 hours to remove bacteria, in order to eliminate the effects of bacterial biomass on scintillation counts and show the incorporation of cellulose-derived carbon into the germ-free worm biomass. A high-throughput antibiotic susceptibility screen of the bacterial strains (including $C$. elegans native gut bacteria and the soil bacteria in Table S1) was conducted to determine an appropriate antibiotic cocktail to eliminate these species from the $C$. elegans gut (Fig. S3). Clearance by the antibiotic cocktail was tested by imaging worms fed with fluorescently labelled Pseudomonas putida (Fig. S4). After clearing the bacterial biomass, worms were mechanically digested in batches (200 worms per sample), and ${ }^{14} \mathrm{C}$ incorporation was measured using scintillation counts. ${ }^{14} \mathrm{C}$ incorporation was significantly higher for $P$. cellulosa colonized

A
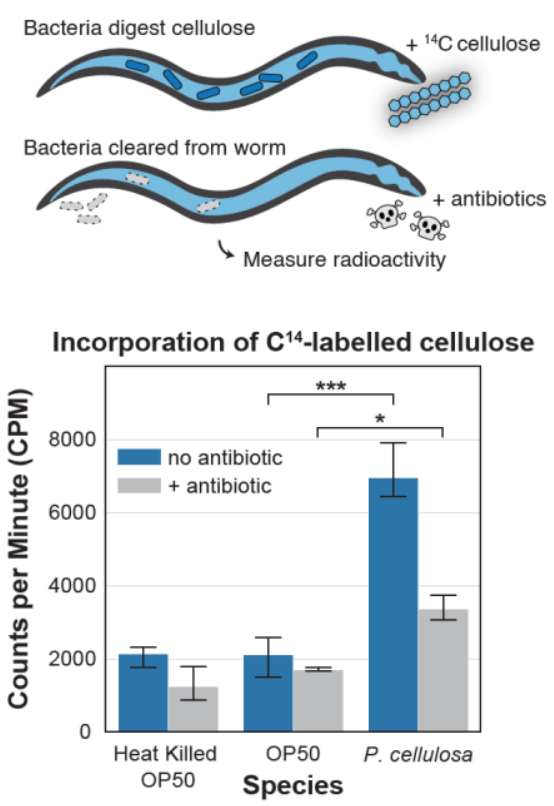

B

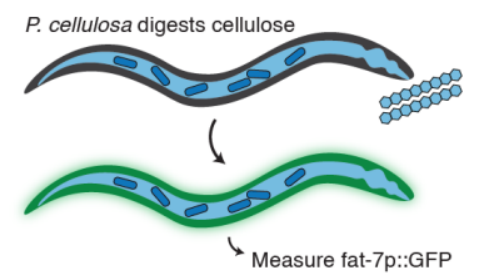

Induction of worm metabolic reporter

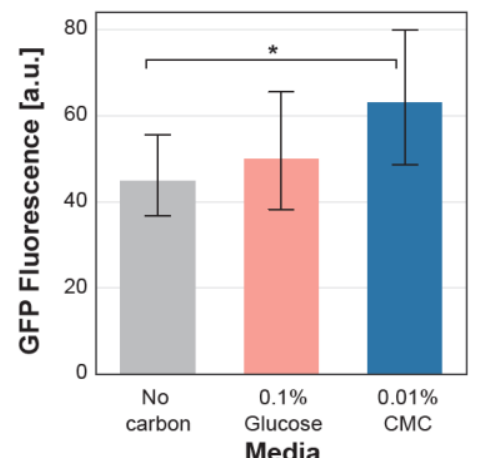

C

Colonize worms with P. cellulosa or OP50

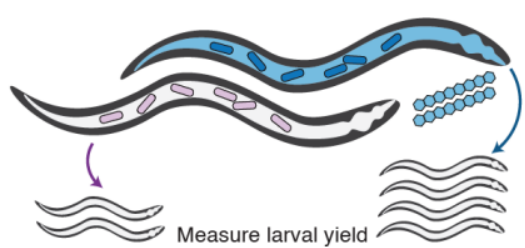

Effect of nutrition on larval yield

- No Carbon $\bullet 0.3 \%$ Glucose $\bullet 0.05 \%$ CMC



Figure 3. Intestinal cellulolytic bacteria allow host utilization of cellulose. A) Incorporation of ${ }^{14} \mathrm{C}$-labelled cellulose. Worms were first colonized with cellulolytic bacteria and then incubated with ${ }^{14} \mathrm{C}$-cellulose to allow carbon utilization. Antibiotic treatment was applied before ${ }^{14} \mathrm{C}$ measurement of worms to eliminate bacterial interference in isotopic reading. ${ }^{14} \mathrm{C}$-cellulose incorporation measurement reflected the isotopic carbon incorporation in C. elegans. B) Induction of the worm fat-7p::GFP metabolic reporter. Adult worms pre-colonized with $P$. cellulosa were incubated in liquid media for 24 hours with the indicated carbon sources, and GFP fluorescence was read in individual worms on a BioSorter large object sorter. C) Effect of nutrition on larval yield. Pre-colonized worms were separated into the individual wells of a 384-well plate, and larvae counts in each well were read after 48 hours. Larval yield comparison indicates the nutrient benefit of cellulolytic activity. $* \mathrm{P}<0.05, * * \mathrm{P}<0.01$, ***P $<0.001$ Student's $\mathrm{t}$ test. In A and B, error bars represent $95 \%$ confidence intervals for the mean. In $\mathrm{C}$, notches represent $95 \%$ confidence intervals for the median. 
worms than for E. coli colonized worms, before and after clearance with antibiotics (Fig. 3A), demonstrating that cellulolytic bacteria in the $C$. elegans gut had hydrolyzed ${ }^{14} \mathrm{C}$ labeled $\mathrm{CMC}$ and that the hydrolyzed glucose had been taken up by the host, as well as by the microbes.

Second, we confirmed that incorporation of carbon from microbiome-digested CMC affected host metabolism by using a fluorescent reporter of host nutritional status (18). We first confirmed that fat-7p::GFP, which is expressed in the intestinal cells of $C$. elegans, was a reliable reporter for carbon uptake by the host, and determined an optimal glucose concentration of $0.1 \%-0.2 \%(\mathrm{w} / \mathrm{v}$ ) for these assays (Fig S5). Young adult wild-type worms (N2 strain, 46 hours after L1 instar) were transferred from plates of live E. coli OP50 + P. cellulosa, where they had been colonized during growth by live bacteria, to $\mathrm{S}$ medium with heat-killed $E$. coli (to provide nitrogen and other nutrients $) \pm$ glucose or CMC. Gentamicin $(10 \mu \mathrm{g} / \mathrm{mL})$ was used to prevent the growth of bacteria outside the worms, ensuring that any nutritional benefit would be conferred by resident bacteria in the gut. Worms pre-colonized with $P$. cellulosa showed an increase in fluorescence from this reporter when provided with CMC as a carbon source (Fig 3B), indicating an increase in available nutrients to the host directly due to the microbial liberation of glucose.

Third, we measured the benefits of carbon incorporation into the new host biomass via larval output, to determine whether colonization by cellulose-degrading bacteria could improve the fitness of the host. Larval output in the worm is a function of nutritional status and can be initiated by adding nutrients after starvation at a specific point in the young adult-mature adult transition (19). We therefore transferred worms from solid media colonization plates to liquid culture supplemented with glucose or CMC 46 hours after plating L1 larvae, to capture worms at the vulnerable point in this transition. Here, worms were placed into individual wells of a 384-well plate, to allow enumeration of larval yield for single adults. After 48 hours in liquid media with a carbon source, the number of larvae produced by individual worms was counted. Worms colonized with $P$. cellulosa, but not with $E$. coli OP50 alone, produced more larvae per adult worm when CMC was provided in the medium (Fig. 3C, S6), again indicating that colonization by the cellulolytic bacterial species allows the worms to benefit from CMC as a carbon source made available by these resident bacteria.

Alongside these efforts, we sought to determine whether adding a co-colonizing species would produce a community with improved function for the host. As an alternative to adding an additional cellulose-degrading bacterium, we sought to take advantage of the probiotic properties of Lactobacillus plantarum, as lactic acid bacteria have previously been shown to have probiotic effects in hosts, including protection against pathogens $(20,21)$. We hypothesized that addition of this probiotic to the gut community could prevent disruption of the community by an intestinal pathogen (here, Salmonella enterica LT2). To determine the effect of L. plantarum on pathogen colonization, worms were pre-colonized with $P$. cellulosa on solid media as previously described, 40 with and without the addition of L. plantarum $\left(10^{9} \mathrm{CFU} /\right.$ plate $)$. After colonization, worms were exposed to $S$. enterica $\left(10^{8} \mathrm{CFU} / \mathrm{mL}\right)$ infection for one hour in liquid culture. Following this initial exposure, worms were washed to remove external pathogens and incubated for 36 hours to allow infection to progress (Fig. 4B). We found that both P. cellulosa and L. plantarum, alone or in 
Figure 4

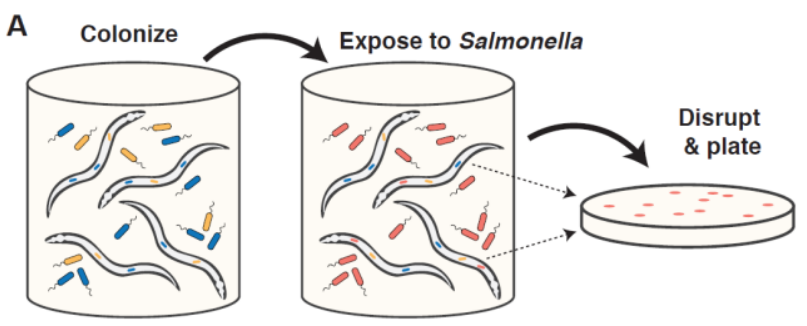

B



Figure 4. Addition of probiotic $L$. plantarum (Lp) protects C. elegans against Salmonella enterica (pathogen) invasion of the gut. Synchronized adult worms were colonized for two days on plates containing E. coli OP50 + P. cellulosa (Pc), E. coli OP50 + Lp, or E. coli OP50 + $\mathrm{Pc}+$ Lp. A) A one-hour exposure to $S$. enterica in liquid culture was used to colonize worms with this pathogen. B) After 36 hours outgrowth of the pathogen, worms pre-colonized with $P$. cellulosa in the presence of $L$. plantarum showed lower pathogen burden than $P$. cellulosa monocolonized worms when CMC was used as the carbon source. Data shown are fold change Salmonella infection (CFU/worm) relative to the $P$. cellulosaonly condition. Error bars represent $95 \%$ confidence interval of the mean. $\mathrm{CMC}=$ $0.01 \%$ carboxymethylcellulose $(\mathrm{w} / \mathrm{v})$. $* \mathrm{P}<$ 0.05 Student's t test.

combination, modestly suppressed pathogen proliferation relative to the control condition (colonization by $E$. coli OP50), but there was no significant difference between the $P$. cellulosa only and the $P$. cellulosa and $L$. plantarum combination conditions without a carbon source.

In the presence of CMC as carbon source, P. cellulosa and L. plantarum in combination suppressed the proliferation of Salmonella by 10-fold compared with what was seen in worms colonized by a single strain (Fig. 4B). These data suggest that the progression of infection in $C$. elegans could be affected by the resident microbiome. What is more, the presence of CMC as a carbon source enhanced the community's capability to suppress pathogens, demonstrating that provision of a specific substrate for the cellulose degrader improved overall community performance. Although there have been other efforts using single-species probiotics to fight against pathogen colonization (22), this work represents a fairly simple microbial community as a way to suppress pathogens in the host intestine. Compared with the single strain system, in a community the individual strains appear to benefit from the complex mix of available nutrients and to acquire an enhanced ability to suppress organisms that are pathogenic to the host.

\section{$\underline{\text { Discussion }}$}

Gut microbe metabolism affects the health of animal hosts in numerous ways, from the liberation of indigestible nutrients (4) to the modification of host-secreted bile acids (7) to the synthesis of certain vitamins and neurotransmitters at significant levels (1). Moreover, metabolic interactions 
among members of the microbiota help maintain community structure, thereby preventing pathogen invasion or other dysbioses (3). C. elegans is a useful model host for microbiome studies, particularly in relation to development and host-pathogen interactions $(9,10)$. In this study, we developed a model of metabolic microbe-host interactions and directly demonstrated that colonization with heterologous bacteria enables $C$. elegans to digest and incorporate carbon from previously indigestible long-chain carbohydrates. In addition to isotopic measurement of carbon incorporation, we demonstrate direct benefits both to the host, with increased larval yield, and to other gut species, which in turn may provide protective effects against pathogens.

We developed assays to quantify the effects of an exogenously introduced microbiome on the nutritional status of $C$. elegans. Datasets collected towards this end include high-throughput in vitro carbon source growth screens to identify bacteria capable of degrading complex carbon sources and in vitro and in vivo screens to identify antibiotic cocktails for removal of colonized bacteria from the $C$. elegans gut. These results can support the use of these organisms as a convenient model for future microbiota-host interaction studies.

Even in these simple systems, good lessons have been learned about the intricacies of interspecies interactions in the potential pitfalls of designing a novel microbiome. First, as we have observed, interactions between microbes must be considered: the introduction of multiple functionally equivalent taxa (as with $P$. cellulosa and $B$. subtilis) does not necessarily increase the functional capacity of the community if there is exploitation competition between strains, which can reduce the functional redundancy that would otherwise be a desirable outcome of this colonization strategy. However, in our experiments, the use of a facultatively interacting taxon $(L$. plantarum) allowed us to add a new functional capacity (resilience against pathogen invasion) by capitalizing on the particular properties of this facultative species. This new function of the community was boosted when we exposed it to CMC, which can be utilized by cellulolytic $P$. cellulosa in the community, demonstrating that distinct bacteria can interact to combat pathogens via cellulose utilization. These results, consistent with other research (23) in this area, indicate that understanding interactions between bacterial strains within an in vivo host environment will be critically important in selecting novel colonists for a host microbial ecosystem. Community assembly starting from modular orthogonal interactions offers a promising approach for designing novel microbiome communities (24-29). Our results have suggested that nutritional interactions can enhance the performance of the system. Recent studies that utilize porphyran as an orthogonal metabolite to enable strain engraftment $(30,31)$ are consistent with this view.

More broadly, with respect to the specific results of these experiments, we believe that further efforts to expand the nutritional scope of animals could have a positive impact on food security. Globally, humans rely on a very slender thread of genetic diversity. In fact, according to the U.N.'s Food and Agriculture Organization (FAO), more than 50\% of all human calories come from just 40 three plants: rice, maize, and wheat (32). Engineering the gut microbiome could permit hosts to digest new food sources or enhance the efficiency of our current nutrition processing capabilities. With rapid progress in enzyme evolution (33) and microbiome engineering (34) techniques, facilitated by developments in model host organisms, engineering the gut microbiome could accelerate nutrient processing in humans or livestock or even expand diets to include entirely new food sources. 


\section{References}

1. T. R. Sampson, S. K. Mazmanian, Control of Brain Development, Function, and Behavior by the Microbiome. Cell Host Microbe. 17, 565-576 (2015).

2. M. J. Barratt, C. Lebrilla, H. Y. Shapiro, J. I. Gordon, The Gut Microbiota, Food Science, and Human Nutrition: A Timely Marriage. Cell Host Microbe. 22, 134-141 (2017).

3. A. B. Shreiner, J. Y. Kao, V. B. Young, The Gut Microbiome in Health and in Disease. Curr. Opin. Gastroenterol. 31, 69-75 (2015).

4. V. Tremaroli, F. Bäckhed, Functional Interactions Between the Gut Microbiota and Host Metabolism. Nature. 489, 242-249 (2012).

5. T. Bourguignon, N. Lo, C. Dietrich, J. Šobotník, S. Sidek, Y. Roisin, A. Brune, T. A. Evans, Rampant Host Switching Shaped the Termite Gut Microbiome. Curr. Biol. 28, 649-654 (2018).

6. B. A. Clemmons, B. H. Voy, P. R. Myer, Altering the Gut Microbiome of Cattle: Considerations of Host-Microbiome Interactions for Persistent Microbiome Manipulation. Microb. Ecol. 77. 523-536(2019).

7. I. Rowland, G. Gibson, A. Heinken, K. Scott, J. Swann, I. Thiele, K. Tuohy, Gut Microbiota Functions: Metabolism of Nutrients and other Food Components. Eur. J. Nutr. 57, 1-24 (2018).

8. J. H. Hehemann, A. G. Kelly, N. A. Pudlo, E. C. Martens, A. B. Boraston, Bacteria of the Human Gut Microbiome Catabolize Red Seaweed Glycans with Carbohydrate-Active Enzyme Updates from Extrinsic Microbes. Proc. Natl. Acad. Sci. U. S. A. 109, 1978619791 (2012).

9. J. Zhang, A. D. Holdorf, A. J. Walhout, C. elegans and its Bacterial Diet as a model for Systems-Level Understanding of Host-Microbiota Interactions. Curr. Opin. Biotechnol. 46, 74-80 (2017).

10. K. T. Jones, K. Ashrafi, Caenorhabditis elegans as an Emerging Model for Studying the Basic Biology of Obesity. Dis. Model. Mech. 2, 224-229 (2009).

11. P. Dirksen, S. A. Marsh, I. Braker, N. Heitland, S. Wagner, R. Nakad, S. Mader, C. Petersen, V. Kowallik, P. Rosenstiel, M. A. Félix, H. Schulenburg, The Native Microbiome of the Nematode Caenorhabditis elegans: Gateway to a New HostMicrobiome Model. BMC Biol. 14, 1-6 (2016).

12. A. A. Houfani, T. Větrovský, P. Baldrian, S. Benallaoua, Efficient Screening of Potential Cellulases and Hemicellulases Produced by Bosea sp. FBZP-16 Using the Combination of Enzyme Assays and Genome Analysis. World J. Microbiol. Biotechnol. 33, 1-6 (2017).

13. R. M. Teather, P. J. Wood, Use of Congo Red-Polysaccharide Interactions in Enumeration and Characterization of Cellulolytic Bacteria from the Bovine Rumen. Appl. Environ. Microbiol. 43, 777-780 (1982).

14. H. Schulenburg, M. A. Félix, The Natural Biotic Environment of Caenorhabditis elegans. Genetics. 206, 55-86 (2017).

15. N. M. Vega, J. Gore, Stochastic Assembly Produces Heterogeneous Communities in the 
Caenorhabditis elegans Intestine. PLoS Biol. 15, 1-20 (2017).

16. K. M. LU, N. C. and Goetsch, Carbohydrate Requirement of Caenorhabditis Elegans and the Final Development of a Chemically Defined Medium. Nematologica. 39, 303-311 (1993).

17. A. Faik, N. J. Price, N. V. Raikhel, K. Keegstra, An Arabidopsis Gene Encoding an $\alpha-$ Xylosyltransferase Involved in Xyloglucan Biosynthesis. Proc. Natl. Acad. Sci. U. S. A. 99, 7797-802 (2002).

18. D. Lee, D. E. Jeong, H. G. Son, Y. Yamaoka, H. Kim, K. Seo, A. A. Khan, T. Y. Roh, D. W. Moon, Y. Lee, S. J. V. Lee, SREBP and MDT-15 Protect C. elegans from GlucoseInduced Accelerated Aging by Preventing Accumulation of Saturated Fat. Genes Dev. 29, 2490-2503 (2015).

19. H. S. Seidel, J. Kimble, The Oogenic Germline Starvation Response in C. elegans. PLoS One. 6, e28074 (2011).

20. B. Bucková, Z. Hurníková, A. Lauková, V. Revajová, E. Dvorožňáková, The AntiParasitic Effect of Probiotic Bacteria via Limiting the Fecundity of Trichinella spiralis Female Adults. Helminthol. 55, 102-111 (2018).

21. T. Ikeda, C. Yasui, K. Hoshino, K. Arikawa, Y. Nishikawa, Influence of Lactic Acid Bacteria on Longevity of Caenorhabditis elegans and Host Defense against Salmonella enterica Serovar Enteritidis. Appl. Environ. Microbiol. 73, 6404-6409 (2007).

22. K. A. B. Kissoyan, M. Drechsler, E. L. Stange, J. Zimmermann, C. Kaleta, H. B. Bode, K. Dierking, Natural C. elegans Microbiota Protects against Infection via Production of a Cyclic Lipopeptide of the Viscosin Group. Curr. Biol. 29, 1030-1037 (2019).

23. E. T. Miller, R. Svanbäck, B. J. M. Bohannan, Microbiomes as Metacommunities: Understanding Host-Associated Microbes through Metacommunity Ecology. Trends Ecol. Evol. 33, 926-935 (2018).

24. J. Friedman, L. M. Higgins, J. Gore, Community Structure Follows Simple Assembly Rules in Microbial Microcosms. Nat. Ecol. Evol. 1, 1-7 (2017).

25. G. L. Medlock, M. A. Carey, D. G. McDuffie, M. B. Mundy, N. Giallourou, J. R. Swann, G. L. Kolling, J. A. Papin, Inferring Metabolic Mechanisms of Interaction within a Defined Gut Microbiota. Cell Syst. 7, 245-257 (2018).

26. A. O. Lopez, N. M. Vega, J. Gore, Interspecies Bacterial Competition Determines Community Assembly in the C. elegans Intestine. bioRxiv. 14, e8157 (2019).

27. O. S. Venturelli, A. C. Carr, G. Fisher, R. H. Hsu, R. Lau, B. P. Bowen, S. Hromada, T. Northen, A. P. Arkin, Deciphering Microbial Interactions in Synthetic Human Gut Microbiome Communities. Mol. Syst. Biol. 14, e8157 (2018).

28. C. Chu, P. B. Adler, Large Niche Differences Emerge at the Recruitment Stage to Stabilize Grassland Coexistence. Ecol. Monogr. 85, 373-392 (2015).

29. W. Kong, D. R. Meldgin, J. J. Collins, T. Lu, Designing Microbial Consortia with Defined Social Interactions. Nat. Chem. Biol. 14, 821-829 (2018). 
Exclusive Metabolic Niche Enables Strain Engraftment in the Gut Microbiota. Nature. 557, 434-438 (2018).

31. S. M. Kearney, S. M. Gibbons, S. E. Erdman, E. J. Alm, Orthogonal Dietary Niche Enables Reversible Engraftment of a Gut Bacterial Commensal. Cell Rep. 24, 1842-1851 (2018).

32. FAO, Fao Statistical Yearbook 2013 (2013; http://www.fao.org/economic/ess/esspublications/ess-yearbook/en/\#.V77pY_krLIU), vol. 1.

33. J. Nielsen, J. D. Keasling, Engineering Cellular Metabolism. Cell. 164, 1185-1197 (2016).

34. N. I. Johns, T. Blazejewski, A. L. C. Gomes, H. H. Wang, Principles for Designing Synthetic Microbial Communities. Curr. Opin. Microbiol. 31, 146-153 (2016).

\section{Acknowledgments:}

The $C$. elegans native microbiome collection was a gift from the Dr. H. Schulenburg group, Christian-Albrechts University, Keil, Germany. Funding: This project was supported by grant HR0011-15-C-0091 from the Defense Advanced Research Projects Agency (DARPA).

\section{Author contributions:}

Q.S., N.M.V., B.C., C.P.M., N.M., M.T., J.J.C., A.S.K., J.G., and T.K.L. designed and conceived the research. Q.S. designed and performed the bacterial CMC degradation capacity quantification assay. C.P.M designed and performed the bacterial CMC utilization assay. Q.S., N.M., and B.C. worked on the bacterial colonization and CMC degradation assay in the worm gut. B.C. conducted the ${ }^{14} \mathrm{C}$-cellulose utilization experiment. N.M.V. performed the worm $f a t-7 p:: G F P$ and larval yield experiments. M.T. and N.M.V. did the community experiment to suppress pathogens. C.P.M. helped with the final data analysis and figure processing.

\section{Conflict of Interest:}

T.K.L. is a co-founder of Senti Biosciences, Synlogic, Engine Biosciences, Tango Therapeutics, Corvium, BiomX, and Eligo Biosciences. T.K.L. also holds financial interests in nest.bio, Ampliphi, IndieBio, MedicusTek, Quark Biosciences, and Personal Genomics. 